\title{
Modeling QCA Defects at Molecular-level in Combinational Circuits
}

\author{
Mariam Momenzadeh, Marco Ottavi, Fabrizio Lombardi \\ Department of Electrical and Computer Engineering, \\ Northeastern University, Boston, MA, 02115 \\ \{mmomenza, mottavi, lombardi@ece.neu.edu\}
}

\begin{abstract}
This paper analyzes the deposition defects in devices and circuits made of Quantum-dot Cellular Automata (QCA) for molecular implementation. Differently from metal-based QCA, in this type of implementation a defect may occur due to the erroneous deposition of cells (made of molecules) on a substrate, i.e. no cell, or an additional cell is placed either near, or within the layout configuration of a QCA device. The effects of an erroneous cell deposition defect are analyzed by considering the induced functional faults for different QCA devices, such as the majority voter, the inverter and various wire configurations (straight, L-shape, coplanar crossing and fanout). Extensive simulation results are provided. As an example, testing of an EXOR circuit is analyzed in detail.
\end{abstract}

Index terms: fault model, defect tolerance, QCA, emerging technology.

\section{Introduction}

As CMOS technology approaches its fundamental physical limits, recent years have seen an extensive research in nanotechnology for manufacturing and assembly of the next generation of integrated circuits (ICs). In an emerging technology, new paradigms arise due to the extremely small feature size and high density of these devices [1]. Among emerging technologies, Quantum-dot Cellular Automata (QCA) [2] [3] relies on new physical phenomena (such as Coulombic interactions), and innovative techniques that radically depart from the CMOS-based model of VLSI. Moreover, QCA not only gives a solution at nano scale, but also it offers a new method of computation and information transformation [4].

Recent developments in cell manufacturing (involving the deposition of molecules on a substrate surface) [11] [13] have substantially changed the nature of QCA process fabrication; in terms of feature size, a QCA cell of few nanometer size has been fabricated through a molecular implementation by a self-assembly process [9]. added: It is anticipated that in these implementations, QCA cells (each made of two dipoles or dots) will be deposited on parallel V-shaped tracks [15]. At this level however, new types of defect are likely to occur. Missing or additional cells are inevitable for molecular implementation, because the process of cell deposition is very sensitive [11]; a small variation in process parameters may result in a defect [13]. Moreover, these defects have pronounced functional effects when they occur either within, or very near to the layout of the target device [10] due to strong cell interactions [14]. So, testing is required for detecting these types of defect in basic QCA devices and circuits. For molecular QCA implementations, multiple defects can be expected; however, as shown in previous papers [10] [5], it is almost impossible to misdetect multiple defects in QCA, i.e. single fault detection is both effective and realistic. For QCA, a defect commonly referred to as the cell misplacement (cell misalignment, presence/absence of a cell) has been shown to have a substantial effect on functionality [10]; the effect of cell misalignment on some QCA devices (mostly applicable to metal implementations) has been studied in [5].

As the logic elements of a QCA-based design are different from CMOS, novel models and related testing schemes are required at device and circuit levels, especially for molecular implementations.

Molecular QCA exhibits different defects from metal QCA. Injection of cell deposition defects on a cartesian layout is required to establish the behavior of QCA-based circuits and to generate appropriate test sets for detection. Although in CMOS only a small percentage of the actual defects 
behaves like stuck-at faults, the stuck-at fault model is still widely used as basis for generating the test set. So in this paper, the behavior of a QCA device in the presence of cell deposition defects is functionally modeled into erroneous logic behavior. We will show that defects in molecular QCA result in unique functional behavior.

The defect characterization of various QCA devices in the presence of a single cell deposition defect and its effects at both device-level and circuit-level are extensively studied. The approach proposed in this work is based on simulating deposition defects (a missing or additional QCA cell) in the layout and investigating their effects at device-level to establish the functional behavior in the presence of such defects. The coherence vector engine of QCADesigner ${ }^{1}$ v1.4.0 is used for simulation.

The coherence vector engine is based on the density matrix approach [12]. Only the effects within the so-called radius of effect $R$ are considered for each cell $i$ using this engine. In all simulation cases, the radius of effect for each cell is set to $40 \mathrm{~nm}$ and all other simulation parameters are set to the default value. Cell size is $10 \times 10 \mathrm{~nm}^{2}$; the cell-to-cell distance and dot size are $2.5 \mathrm{~nm}$. Variations in these parameters have been previously investigated in [14].

The rest of this paper is organized as follows. Section 2 deals with a brief review of QCA; Section 3 presents the assumed defect model for cell deposition in a QCA molecular implementation. Sections 4, 5 and 6 analyze in detail the defects in QCA devices. The analysis of these results with respect to the faults caused by the defects is provided in Section 7. Section 8 extends the device-level analysis to an EXOR circuit implemented by QCA. Conclusions are addressed in the last Section.

\section{Review of QCA}

A QCA cell can be viewed as a set of four charge containers or "dots", positioned at the corners of a square. The cell contains two extra mobile electrons which can quantum mechanically tunnel between dots, but not cells. The electrons are forced to the corner positions by Coulomb repulsion.

Therefore electrons have a preferential alignment along one of the two perpendicular cell axes, as shown in Figure 1. The polarization $P$ measures the extent of this alignment. If the charge on a $\operatorname{dot} i$ is $\rho_{i}$, then the polarization is defined [6] as

$$
P=\frac{\left(\rho_{1}+\rho_{3}\right)-\left(\rho_{2}+\rho_{4}\right)}{\rho_{1}+\rho_{2}+\rho_{3}+\rho_{4}}
$$

If the two extra electrons are completely localized on dots 1 and 3 , the polarization is +1 (binary 1 ); if they are localized on dots 2 and 4 , the polarization is - 1 (binary 0 ). The tunneling between dots implies that $\rho_{i}$ may not be integers as well as polarization values.

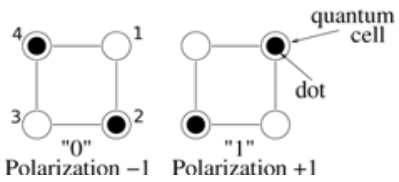

Figure 1. QCA cell and polarization states

Unlike conventional logic circuits in which information is transferred by electrical current, QCA operates by the Coulombic interaction that connects the state of one cell to the state of its neighbors. This results in a technology in which information transfer (interconnection) is the same as information transformation (logic manipulation) with low power dissipation [6].

One of the basic logic gates in QCA is the so-called majority voter (MV) with logic function $\operatorname{Maj}(A, B, C)=A B+A C+B C$. MV can be realized by 5 QCA cells, as shown in Figure 2(b). Logic AND and OR functions can be implemented from the MV by setting an input (the so-called programming or control input) permanently to a "0" or " 1 " value. The inverter (INV) is the other basic gate in QCA and is shown in Figure 2(a). The binary wire and inverter chain (as interconnect fabric) are shown in Figure 2(c)(d).

In VLSI systems, timing is controlled through a reference signal (i.e. the clock), however timing in QCA is accomplished by clocking in four distinct and periodic phases [7] (Figure 3). A QCA circuit is partitioned into serial (one-dimensional) zones, and each zone is maintained in a phase.

\footnotetext{
${ }^{1}$ QCADesigner is developed by the ATIPS lab at the University of Calgary in Canada. 


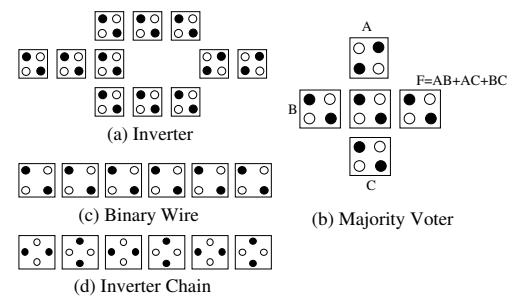

Figure 2. QCA cell and polarization states

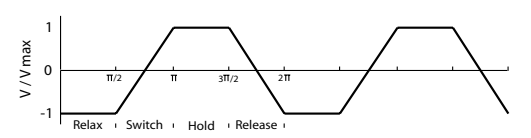

Figure 3. Cmos-Clock-Signal

\section{Defect Model}

A model which characterizes defects in QCA devices and circuits, must consider [14] the unique fabrication and assembly processes of this technology at molecular level. According to [8], in the present stage of QCA manufacturing, defects are possible in both the synthesis phase, in which the individual cells (molecules) are manufactured, and the deposition phase in which the cells are placed in a specific location on the surface. Manufacturing defects may cause a cell to have missing or extra dots or/and electrons. These defects are fatal to the correct operation of the cell and easy to detect. However, defects are much more likely to occur in the deposition process than in the synthesis process. These defects are usually categorized as cell misplacement. A missing dot (or additional dot) is very unlikely due to the ease of purification of small inorganic molecules [8]. For example, Nuclear Magnetic Resonance (NMR) has an estimated minimum purity of $99 \%$ for model compounds such as the Creutz-Taube (CT) Ion (a 2-dot model or dipole for half of a cell). Moreover, electrochemical measurements for the CT Ion have shown that fewer than one molecule in $10^{5}$ are in the incorrect charge state [13]. Yet placing the individual cells during deposition is difficult, various types of cell misplacement may occur (such as cell misalignment, missing cell, additional cell, etc.). The effects of geometry misalignment on metal cells of some QCA devices have been studied in [5].

Molecular QCA presents unique challenges: bonding of the array surface requires complexes by spectroscopic and electrochemical techniques [11]; moreover, the presence of strongly bound, chemically robust, mixed valence complexes in the required chemistry has been extensively treated. Perturbation of the chemical complex by surface binding using a gold electrode by an electrochemical method has been investigated resulting in an assembly of biased, vertically oriented two-dot structures (dipole) sandwiched between two electrodes [11]. The assembly of a symmetric square cell containing two ferrocene and two ferrocenium moieties with measured properties that make it suitable as a component for charge-coupled QCA circuits, has been shown in [13]. However, deposition defects are still widely reported and they must be carefully considered because they may affect the correct operation of QCA circuits. This has a stronger influence when a cell deposition defect occurs either within or very near to a device, thus creating unwanted interactions [14].

In this paper the following types of single cell deposition defect are investigated for a molecular implementation of QCA:

1) An extra or additional cell $\left(D_{A}\right)$ is a defect in which an additional cell is deposited on the substrate; this extra cell is erroneously deposited along the device perimeter (adjacency boundary) of the original (defect-free) configuration.

2) In a missing cell deposition defect $\left(D_{M}\right)$, a particular cell is missing in the original (defect-free) configuration of the device or circuit.

For $D_{A}$, the adjacency boundary of a cell deposition defect is considered in this paper: the adjacency boundary consists of the area around the cell perimeter of a device or circuit in which the presence of a defect due to an additional cell deposition may occur. The proximity (one cell distance) of $D_{A}$ to the QCA device causes strong interaction to be present as it modifies the device layout itself. This defect model can be used to characterize faults in molecular QCA. 


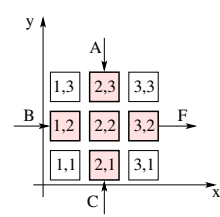

(a)

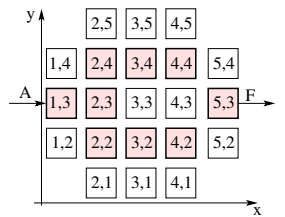

(b)

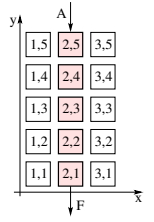

(c)

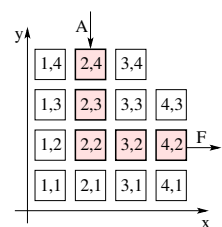

(d)

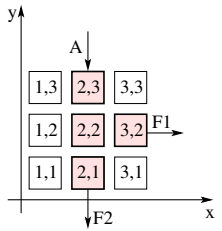

(e)

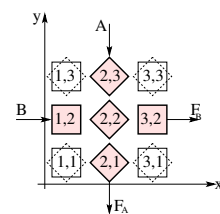

(f)

Figure 4. Considered QCA devices: (a) Majority Voter (b) Inverter (c) Straight-wire (d) L-shaped wire (e) Fanout (f) Coplanar Crossing

In QCA, the set of basic devices is given by the Majority Voter, the Inverter, and the Wire (in different configurations such as Straight, L-Shaped, Coplanar Crossing and Fanout). In the assumed model for molecular deposition, the defect set is given by an additional (or extra) cell or a missing cell; the fault set is made of the functional faults resulting from the occurrence of a defect (in the following sections it will be shown that this set is given by stuck-at (such as S-a- $A$, S-a- $A^{\prime}$, S-a$\left.B^{\prime}\right)$, different output function ( $\operatorname{such}$ as $\operatorname{Maj}\left(A^{\prime}, B, C^{\prime}\right)$ ) and undet, where undet refers to the state of undetermined QCA polarization (either extremely low polarization, or presence of glitches in a signal) and denoted by "-". As for VLSI, a defect may give rise to only few faults; this depends on the geometry of the device as well as the interactions among QCA cells [10].

In the next Sections, the fault set of different QCA devices shown in Figure 4 are established through extensive simulation and the impact of the defects is evaluated. Input and output cells are located outside the layout of each device and a single missing or additional cell deposition defect is injected either in the original device, or along the adjacency boundary, i.e. no defect is injected for input/output cells.

\section{Majority Voter}

The first device analyzed in this paper, is the Majority Voter, MV. Figure 4 (a) shows the cell layout of the MV and the locations of the possible cell deposition defects. The $x$ and $y$ coordinates are used to identify the cells in the cartesian layout. Simulation results are reported in Table 1. $D_{M}$ shows the coordinates of the missing cell deposition defect; $D_{A}$ shows the coordinates of the extra cell deposition defect; $P_{\max }$ denotes the highest magnitude polarization of $F$. This depicts the strength of the signal between the highest polarization (1) and an extremely low polarization (0). All input values are applied with maximum polarization. Remarkably, an extra cell deposition never affects the output as a majority function. As for the missing cell deposition defect, the following considerations are valid: (1) A missing deposition defect of cell $(2,1)$ or $(2,3)$ leads in both cases to $F=B$ which confirms previous results found in the technical literature [10]. (2) A missing deposition defect of the middle cell $(2,2)$ results in a majority function in which the input signals $A$ and $C$ are inverted, i.e. $\operatorname{Maj}\left(A^{\prime}, B, C^{\prime}\right)$.

\begin{tabular}{|c||c|c|}
\hline$D_{M}$ & $F$ & $P_{\max }$ \\
\hline 2,1 & $B$ & .95 \\
\hline 1,2 & $\operatorname{Maj}(A, B, C)$ & .95 \\
\hline 2,3 & $B$ & .95 \\
\hline 2,2 & $\operatorname{Maj}\left(A^{\prime}, B, C^{\prime}\right)$ & .95 \\
\hline 3,2 & - & .09 \\
\hline \hline$D_{A}$ & $F$ & $P_{\max }$ \\
\hline 1,1 & $\operatorname{Maj}(A, B, C)$ & .95 \\
\hline 1,3 & $\operatorname{Maj}(A, B, C)$ & .95 \\
\hline 3,1 & $\operatorname{Maj}(A, B, C)$ & .95 \\
\hline 3,1 & $\operatorname{Maj}(A, B, C)$ & .95 \\
\hline
\end{tabular}

\section{Table 1. Simulation results for MV}

\section{Inverter}

The second QCA device analyzed in this manuscript, is the Inverter (INV). Figure 4 (b) shows the layout of the inverter as well as the locations of possible defects; the simulation results are reported 
in Table 2. The extra cells $(5,2)$ and $(5,4)$ and the middle cell $(4,3)$ change the actual layout of this device to a fanout/fanin structure, thus the output is not inverted i.e. $F=A$. For a $D_{M}$ the following considerations apply: (1) A missing deposition defect at $(2,3)$ or $(2,4)$ or $(2,2)$ results into $F=A$. For cell $(2,3)$ this is a rather obvious condition, because it generates a concatenation of two inverters; for the other cells it appears that the double inverted path has a stronger effect on the output than the correct path. (2) When cell $(5,3)$ is not deposited, the output is isolated taking an undetermined value.

\begin{tabular}{|c|c|c||c|c|c|}
\hline$D_{M}$ & $F$ & $P_{\max }$ & $D_{A}$ & $F$ & $P_{\max }$ \\
\hline \hline 2,2 & $A$ & .95 & 2,1 & $A^{\prime}$ & .95 \\
\hline 3,2 & $A^{\prime}$ & .95 & 3,1 & $A^{\prime}$ & .95 \\
\hline 4,2 & $A^{\prime}$ & .95 & 4,1 & $A^{\prime}$ & .95 \\
\hline 1,3 & $A^{\prime}$ & .95 & 1,2 & $A^{\prime}$ & .95 \\
\hline 2,3 & $A$ & .95 & 5,2 & $A$ & .92 \\
\hline 5,3 & - & 0 & 3,3 & $A^{\prime}$ & .95 \\
\hline 2,4 & $A$ & .95 & 4,3 & $A$ & .95 \\
\hline 3,4 & $A^{\prime}$ & .95 & 1,4 & $A^{\prime}$ & .95 \\
\hline 4,4 & $A^{\prime}$ & .95 & 5,4 & $A$ & .92 \\
\hline & & & 2,5 & $A^{\prime}$ & .95 \\
\hline & & & 3,5 & $A^{\prime}$ & .95 \\
\hline & & & 4,5 & $A^{\prime}$ & .95 \\
\hline
\end{tabular}

Table 2. Simulation results for Inverter

\section{Wire}

In this section, the simulation results for the wire configurations and related arrangements (straight, L-shaped, fanout and coplanar crossing) are presented.

\subsection{Straight Wire}

A straight wire of five cell length is shown in Figure 4 (c) together with the possible defect locations in the adjacency boundary. The simulation results are reported in Table 3 (a). These results show that the straight wire is not sensitive to an additional cell defect as in all cases $F=A$. Moreover, also for a single missing cell deposition defect, $F=A$.

\begin{tabular}{|c||c|c|}
\hline$D_{M}$ & $F$ & $P_{\max }$ \\
\hline 2,5 & $A$ & .95 \\
\hline 2,4 & $A$ & .95 \\
\hline 2,3 & $A$ & .95 \\
\hline 2,2 & $A$ & .95 \\
\hline 2,1 & $A$ & .95 \\
\hline \hline$D_{A}$ & $F$ & $P_{\max }$ \\
\hline 3,5 & $A$ & .95 \\
\hline 3,4 & $A$ & .95 \\
\hline 3,3 & $A$ & .95 \\
\hline 3,2 & $A$ & .95 \\
\hline 3,1 & $A$ & .93 \\
\hline \hline$D_{A}$ & $F$ & $P_{\max }$ \\
\hline 1,5 & $A$ & .95 \\
\hline 1,4 & $A$ & .95 \\
\hline 1,3 & $A$ & .95 \\
\hline 1,2 & $A$ & .95 \\
\hline 1,1 & $A$ & .93 \\
\hline
\end{tabular}

(a)

\begin{tabular}{|c||c|c|}
\hline$D_{M}$ & $F$ & $P_{\max }$ \\
\hline 2,4 & $A$ & .95 \\
\hline 2,3 & $A$ & .95 \\
\hline 2,2 & $A^{\prime}$ & .95 \\
\hline 3,2 & $A$ & .95 \\
\hline 4,2 & $A$ & .10 \\
\hline \hline$D_{A}$ & $F$ & $P_{\max }$ \\
\hline 1,4 & $A$ & .95 \\
\hline 1,3 & $A$ & .95 \\
\hline 1,2 & $A$ & .95 \\
\hline 1,1 & $A$ & .95 \\
\hline 2,1 & $A$ & .95 \\
\hline \hline$D_{A}$ & $F$ & $P_{\max }$ \\
\hline 3,1 & $A$ & .95 \\
\hline 4,1 & $A$ & .93 \\
\hline 3,4 & $A$ & .95 \\
\hline 3,3 & $A$ & .95 \\
\hline 4,3 & $A$ & .93 \\
\hline \multicolumn{3}{|c|}{$(\mathrm{b})$} \\
\hline
\end{tabular}

(b)

Table 3. Simulation Results for Wire (a) Straight (b) L-Shaped

\subsection{L-shaped Wire}

The L-shaped wire is considered next; this type of wire is shown in Figure 4 (d). The simulation results are reported in Table 3 (b). The additional cell deposition defect does not affect the output 
value $F=A$, while a missing deposition defect of a cell due to an erroneous deposition has an effect only if it is the corner cell $(2,2)$, i.e. in this last case, the wire behaves as an inverter $\left(F=A^{\prime}\right)$.

\subsection{Fanout Wire}

A fanout wire allows to duplicate a signal, so it is part of the set of basic routing devices that must be characterized. The considered layout is shown in Figure 4 (e); the locations of defects are also shown. Due to symmetry, the results of Table 4 are valid for any cell rotation of the reported layout. The results show that an extra cell deposition defect causes no functional fault in any of the output branches; instead the missing cell defect causes the output to take an undetermined value. This occurs when the cell affected by the defect is at the closest distance, (i.e. cells $(2,1)$ and $(3,2))$; if the affected cell is the middle cell $(2,2)$, then an inverter is formed on the path to $F 1$ and therefore, an erroneous output is generated.

\begin{tabular}{|c||c|c|c|}
\hline$D_{M}$ & $F 1$ & $F 2$ & $P_{\max }$ \\
\hline 2,1 & $A$ & - & $.96, .06$ \\
\hline 2,2 & $A^{\prime}$ & $A$ & $.95, .95$ \\
\hline 3,2 & - & $A$ & $.01, .95$ \\
\hline 2,3 & $A$ & $A$ & $.95, .95$ \\
\hline \hline Added Cell & $F 1$ & $F 2$ & Max. Pol. \\
\hline$D_{A}$ & $A$ & $A$ & $P_{\max }$ \\
\hline 3,1 & $A$ & $A$ & $.92, .93$ \\
\hline 1,2 & $A$ & $A$ & $.95, .95$ \\
\hline 1,3 & $A$ & $A$ & $.95, .95$ \\
\hline 3,3 & $A$ & $A$ & $.92, .95$ \\
\hline
\end{tabular}

\section{Table 4. Simulation Results for Fanout Wire}

\subsection{Coplanar Wire Crossing}

The last device which is considered in this paper, is the so-called coplanar crossing of two QCA wires. The simulation results for the wire crossing device are given in Table 5. The extra cell deposition has been considered in both the rotated and non rotated cell arrangements.

From the results reported in the tables, the following considerations can be drawn: (1) Extra non-rotated cells do not affect the correct outputs, while extra rotated cells affect one output only. (2) A missing cell always causes a faulty output. Moreover, two types of functional fault may occur: inversion of a signal and interference (an erroneous routing takes place in the coplanar crossing). Note that the simultaneous occurrence of these two types of fault is also possible.

\section{Fault Analysis}

The functional effects of the deposition defects permit to define a fault set for each QCA device, as related to the impact of its occurrence. Where the impact of a defect type $\left(D_{A}\right.$ or $\left.D_{M}\right)$ is defined

\begin{tabular}{|c||c|c|c|}
\hline$D_{M}$ & $F_{A}$ & $F_{B}$ & $P_{\max }$ \\
\hline 2,1 & $A^{\prime}$ & $B$ & $.11, .95$ \\
\hline 1,2 & $A$ & $A^{\prime}$ & $.97, .95$ \\
\hline 2,2 & $A^{\prime}$ & $B$ & $.97, .95$ \\
\hline 3,2 & $A$ & - & $.97, .01$ \\
\hline 2,3 & $A^{\prime}$ & $B$ & $.97, .95$ \\
\hline \hline$D_{A}$ as Non-rotated Cell & $F_{A}$ & $F_{B}$ & $P_{\max }$ \\
\hline 1,1 & $A$ & $B$ & $.97, .95$ \\
\hline 3,1 & $A$ & $B$ & $.97, .93$ \\
\hline 1,3 & $A$ & $B$ & $.97, .95$ \\
\hline 3,3 & $A$ & $A^{\prime}$ & $.97, .93$ \\
\hline \hline$D_{A}$ as Rotated Cell & $F_{A}$ & $F_{B}$ & $P_{\max }$ \\
\hline 1,1 & $A$ & $B$ & $.98, .95$ \\
\hline 3,1 & $A$ & $B$ & $.98, .95$ \\
\hline 1,3 & $A$ & $B$ & $.97, .95$ \\
\hline 3,3 & $A$ & $B$ & $.97, .95$ \\
\hline
\end{tabular}


as the number of those defects resulting in functional faults. For example, the results reported in the previous sections have shown that the effect of an extra cell deposition is mostly ineffective at the output apart from the cases of the inverter and the coplanar wire crossing.

Table 6 provides the fault set obtained from the above reported simulations for each of the considered devices. It can be noticed that:

1) For MV, the impact of missing cell defects is higher than for additional cell deposition defects, i.e. for the MV $80 \%$ of the missing cell defects cause a functional fault at the output while no additional cell deposition defect produces an erroneous output.

2) For the QCA inverter, most of the defects result in a functional fault of a non-inverted output (85.7\%) type. The impact of $D_{M}$ is relatively higher than $D_{A}$ accounting for 4 faulty outputs out of 9 possible defect sites (33\%) versus 3 faulty outputs out of 12 possible defect sites (25\%).

3) The Straight Wire shows excellent resilience to single cell deposition defects as the output is never affected.

4) For the L-Shaped Wire, the extra cell deposition defect generates no functional fault, while the missing cell deposition defect causes just a single fault. In all cases its impact is very low.

5) For the Fanout Wire, the extra cell causes no fault; however, a missing cell deposition defect causes an erroneous output for $50 \%$ on $F 1$ and $25 \%$ on $F 2$.

6) For the Coplanar Wire Crossing, the extra cell deposition defect causes a fault occurrence, while the missing cell deposition defect causes always an erroneous $F_{B}$ and it results in an erroneous $F_{A}$ for $66 \%$ of the cases.

\begin{tabular}{|c|c|c|c|}
\hline Device & $\begin{array}{c}D_{A} \\
\text { Impact }(\%)\end{array}$ & $\begin{array}{c}D_{M} \\
\text { Impact }(\%)\end{array}$ & Fault Set \\
\hline$\overline{\mathrm{MV}}$ & $\overline{0}$ & 80 & $\begin{array}{c}\text { S-a- } B \\
M a j\left(A^{\prime}, B, C^{\prime}\right) \\
\text { undet }\end{array}$ \\
\hline$\overline{\text { INV }}$ & 25 & 33 & $\begin{array}{l}\text { S-a- } A \\
\text { undet }\end{array}$ \\
\hline $\begin{array}{l}\text { STRAIGHT } \\
\text { WIRE }\end{array}$ & 0 & 0 & \\
\hline $\begin{array}{l}\text { L-SHAPED } \\
\text { WIRE }\end{array}$ & 0 & 20 & S-a- $A^{\prime}$ \\
\hline $\begin{array}{l}\text { FANOUT } \\
\text { WIRE }\end{array}$ & $\begin{array}{l}0(\mathrm{~F} 1) \\
0(\mathrm{~F} 2)\end{array}$ & $\begin{array}{l}50(\mathrm{~F} 1) \\
25(\mathrm{~F} 2)\end{array}$ & $\begin{array}{l}\text { S-a- } A^{\prime} \\
\text { undet } \\
\text { undet }\end{array}$ \\
\hline $\begin{array}{l}\text { COPLANAR } \\
\text { WIRE } \\
\text { CROSSING }\end{array}$ & $\begin{array}{c}0\left(\mathrm{~F}_{A}\right) \\
12.5\left(\mathrm{~F}_{B}\right)\end{array}$ & $\begin{array}{l}66\left(\mathrm{~F}_{A}\right) \\
40\left(\mathrm{~F}_{B}\right)\end{array}$ & $\begin{array}{cl}\mathrm{S}-\mathrm{a}-A^{\prime} \\
\mathrm{S}-\mathrm{a}-A^{\prime} & \text { (interference) } \\
\text { undet }\end{array}$ \\
\hline
\end{tabular}

Table 6. Fault set and defect impact for QCA devices

\section{Case Study: Test vector generation of an EXOR Circuit}

From the above obtained fault set has been applied to generate the test vector applicable to a 2 inputs EXOR circuit. The QCA schematic of the considered EXOR gate circuit is shown in Figure 5. Also in the QCA case, fault activation and propagation are taken into account for testing. This data has been validated by a preliminary step in which injection of single deposition defects has been performed on the QCA circuit and the value at the primary outputs (PO) has been compared with the one obtained by combining the effects of the fault set on each of the devices in the circuit. This process allows to validate and confirm that the device-level analysis presented in previous sections can be extended to circuit-level, while obtaining consistent results for the functional fault set. In this respect, full validation has been accomplished; an interesting effect which has been captured for molecular QCA, is the undetermined fault. These faults can be propagated and detected only

Proceedings of the 2005 20th IEEE International Symposium on Defect and Fault Tolerance in VLSI Systems (DFT'05) 


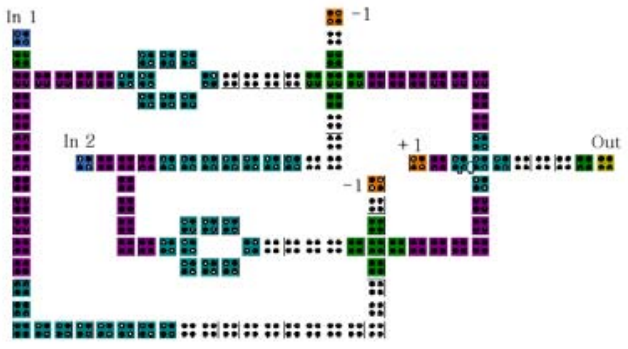

(a)

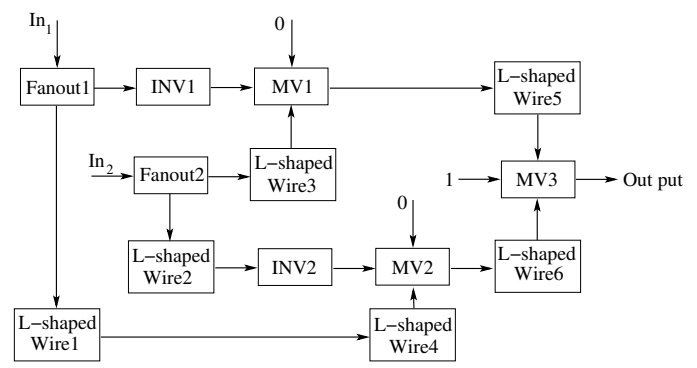

(b)

Figure 5. EXOR gate circuit, (a) QCA layout (b) Device level schematic

when the output of the affected device is a primary output: when a device that is affected by this type of fault is located internally to the QCA circuit, the undetermined value never propagates and the correct output is always observed at the primary output. This is caused by the regenerative effect of the non-linear nature of the cell-to-cell QCA response, as a weak polarization appears only at an isolated output; however, when the output is not isolated, cell-to-cell interaction causes the regeneration of the weak polarization and therefore propagation of the functional fault does not occur. An undermined fault that is non propagated, is denoted as $N P_{\text {undet }}$. Table 7 shows all functional faults affecting the devices in the EXOR circuit given in schematic form in Figure 5 (b). Test vectors are reported; the undetermined fault at MV3 is labeled as non propagating conditional fault (denoted as $N P C_{\text {undet }}$ ). This refers to the condition whether MV3 provides the PO of the QCA circuit. If so, then the undetermined fault is detected; else, (i.e. for example a QCA wire is placed after the output of MV3), then this fault is $N P_{\text {undet }}$.

\begin{tabular}{|c|c|c|c|}
\hline Fault Site & Fault & $\begin{array}{l}\text { test vector } \\
I n_{1} I n_{2}\end{array}$ & $\begin{array}{c}\text { PO value } \\
\text { fault-free (faulty) }\end{array}$ \\
\hline \multirow[t]{3}{*}{ 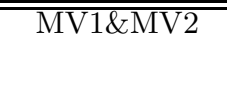 } & $\overline{\mathrm{S}-\mathrm{a}-B}$ & $\overline{000}$ & cl(1) \\
\hline & $\operatorname{Maj}\left(A^{\prime}, B, C^{\prime}\right)$ & 00 & $0(1)$ \\
\hline & undet & $N P_{\text {undet }}$ & $I n_{1} \oplus I n_{2}\left(I n_{1} \oplus I n_{2}\right)$ \\
\hline \multirow[t]{3}{*}{ MV3 } & $\mathrm{S}-\mathrm{a}-B$ & 00,11 & $0(1)$ \\
\hline & $\overline{M a j}\left(A^{\prime}, B, C^{\prime}\right)$ & 00,11 & $0(1)$ \\
\hline & undet & $N P C_{\text {undet }}$ & \\
\hline INV1 & S-a- $A$ & $I n_{1} 1$ & $I n_{1}^{\prime}\left(I n_{1}\right)$ \\
\hline INV2 & $\mathrm{S}-\mathrm{a}-A$ & $1 I n_{2}$ & $I n_{2}^{\prime}\left(\operatorname{In}_{2}\right)$ \\
\hline \multirow[t]{3}{*}{ Fanout1 } & $\mathrm{s}-\mathrm{a}-A^{\prime}$ for $f_{1}$ & $I n_{1} 1$ & $\operatorname{In}_{1}^{\prime}\left(\operatorname{In}_{1}\right)$ \\
\hline & undet $f_{1}$ & $N P_{\text {undet }}$ & $I n_{1} \oplus I n_{2}\left(I n_{1} \oplus I n_{2}\right)$ \\
\hline & undet $f_{1}$ & $N P_{\text {undet }}$ & $\operatorname{In}_{1} \oplus \operatorname{In} n_{2}\left(I n_{1} \oplus I n_{2}\right)$ \\
\hline \multirow[t]{3}{*}{ Fanout2 } & S-a- $A^{\prime}$ for $f_{1}$ & $1 I n_{2}$ & $\operatorname{In}_{2}^{\prime}\left(I n_{2}\right)$ \\
\hline & undet $f_{1}$ & $N P_{\text {undet }}$ & $I n_{1} \oplus I n_{2}\left(I n_{1} \oplus I n_{2}\right)$ \\
\hline & undet $f_{2}$ & $N P_{\text {undet }}$ & $\operatorname{In}_{1} \oplus \operatorname{In} n_{2}\left(I n_{1} \oplus \operatorname{In} n_{2}\right)$ \\
\hline L-shaped wire1 & $\mathrm{S}-\mathrm{a}-A^{\prime}$ & $\operatorname{In}_{1} 0$ & $\operatorname{In}_{1}\left(\operatorname{In}_{1}^{\prime}\right)$ \\
\hline L-shaped wire2 & S-a- $A^{\prime}$ & $1 I n_{2}$ & $\operatorname{In}_{2}^{\prime}\left(I n_{2}\right)$ \\
\hline L-shaped wire3 & $\mathrm{S}-\mathrm{a}-A^{\prime}$ & $0 I n_{2}$ & $I n_{2}\left(I_{2}^{\prime}\right)$ \\
\hline L-shaped wire4 & $\mathrm{S}-\mathrm{a}-A^{\prime}$ & $\operatorname{In}_{1} 0$ & $\operatorname{In}\left(\operatorname{In}_{1}^{\prime}\right)$ \\
\hline L-shaped wire5 & $\mathrm{S}-\mathrm{a}-A^{\prime}$ & $I n_{1} 1,00$ & $\operatorname{In}_{1}^{\prime}\left(\operatorname{In}_{1}\right), 0(1)$ \\
\hline L-shaped wire6 & $\mathrm{S}-\mathrm{a}-A^{\prime}$ & $1 I n_{2}, 00$ & $\operatorname{In}_{2}^{\prime}\left(\operatorname{In}_{2}\right), 0(1)$ \\
\hline
\end{tabular}

\section{Table 7. Test vectors for EXOR gate circuit}

\section{Conclusion}

This paper has characterized and analyzed the deposition defects in devices and circuits made of Quantum-dot Cellular Automata (QCA) for molecular implementation. Differently from metalbased QCA, in this type of implementation a defect may occur due to the erroneous deposition of cells on a substrate, i.e. no cell, or an additional cell is placed either near or within the layout 
configuration of a QCA device. The effects of an erroneous cell deposition defect have been analyzed by considering the induced functional faults for different QCA devices, such as the majority voter, the inverter and various wire configurations (straight, L-shape, coplanar crossing and fanout). It has been shown that for an MV, a single missing cell defect has a stronger effect than a single additional cell deposition defect. For the QCA inverter, most of the single deposition defects result in a functional fault of a non-inverted output type, confirming that also in this device, missing cell deposition defects have pronounced effects. As for the QCA wire, the straight configuration has been shown to have excellent resilience to single cell deposition defects. This feature is also applicable to the L-shaped wire: the extra cell deposition defect generates no functional fault, while the missing cell deposition defect causes just a single fault. For the fanout and coplanar crossing configurations, the extra cell causes at most a fault; however, a missing cell deposition defect results in different faults at the two outputs. Extensive simulation results have been provided for defect characterization. An interesting effect that has been observed in molecular QCA, is the so-called undetermined fault (lack of polarity or presence of glitches in a signal). It has been shown that this type of fault can be propagated and detected only when the output of the affected device is a primary output, i.e. when a device that is affected by this type of fault is located internally to the QCA circuit, the undetermined value never propagates and the correct output is always observed at the primary output. This is caused by the regenerative effect of the non-linear nature of the cell-to-cell QCA response, as a weak polarization appears only at an isolated output; however, when the output is not isolated, cell-to-cell interaction causes the regeneration of the weak polarization and therefore the non propagation of the functional fault occurs.

\section{References}

[1] R. Compano, L. Molenkamp, D.J. Paul, "Technology Roadmap for Nanoelectroincs", European Commission IST programme, Future and Emerging Technologies.

[2] C.S. Lent, P.D. Tougaw and W. Porod, "Quantum Cellular Automata: The Physics of Computing with Arrays of Quantum Dot Molecules", PhysComp '94: Proceedings of the Workshop on Physics and Computing, IEEE Computer Society Press, pp. 5-13, 1994.

[3] C.G. Smith, "Computation Without Current", Science, vol 284, pp. 274, 1999.

[4] A.O. Orlov, I. Amlani, G.H. Bernstein, C.S. Lent and G.L. Snider, "Realization of a Functional Cell for QuantumDot Cellular Automata", Science, vol. 277, pp 928-930, 1997.

[5] M.B. Tahoori, M. Momenzadeh, J. Huang, F. Lombardi, "Defects and Faults in Quantum Cellular Automata at Nano Scale", VLSI Test Symposium, pp. 291-296, 2004.

[6] P.D. Tougaw and C.S. Lent, "Logical Devices Implemented Using Quantum Cellular Automata", Journal of Applied Physics, vol 75(3), pp. 1818-1825, 1994.

[7] K. Hennessy and C.S. Lent, "Clocking of Molecular Quantum-Dot Cellular Automata", Journal of Vaccum Science and Technology, vol. 19(5), pp. 1752-1755, 2001.

[8] Personal communication with Professor Marya Lieberman, Department of Chemistry and Biochemistry, University of Notre Dame, IN, USA.

[9] C.S. Lent, B. Isaksen and M. Lieberman, "Molecular Quantum-Dot Cellular Automata", Journal of the American Chemical Society, vol. 125(4), pp. 1056-1063, 2003.

[10] M.B. Tahoori, M. Momenzadeh, J. Huang and F. Lombardi, "Testing of Quantum Cellular Automata," IEEE Transactions on Nanotechnology, vol. 3, no. 4, pp. 432-442, 2004.

[11] H. Qi, S. Sharma, Z. Li, G.L. Snider, A.O. Orlov, C.S. Lent and T. P. Fehiner, "Molecular Quantum Cellular Automata Cells: Electric Field Driven Switching of a Silicon Surface Bound Array of Vertically Oriented TwoDot Molecular QCA," Journal of the Am. Chem. Society, (JACS Articles) vol. 125, no. 49, pp. 15250-15259, 2003.

[12] QCADesigner Web Page : www.qcadesigner.ca/manual/coherence_vector_engine.html

[13] J. Jiao, G.L. Long, F. Grandjean, A.M. Beatty and T.P. Fehiner, "Building Blocking for the Molecular Expression of QCA, Isolation and Characterization of a Covalently Bounded Square Array of two Ferrocenium and Two Ferrocene Complexes," Journal of the Am. Chem. Society (JACS Communications) vol. 125, no. 25, pp. 7522$7523,2003$.

[14] J. Huang, M. Momenzadeh, M.B. Tahoori and F. Lombardi, "Defect Characterization for Scaling of QCA Devices," Proc. IEEE International Symposium on DFT in VLSI Systems, pp. 30-38, 2004.

[15] W. Hu, K. Sarveswaran, M. Lieberman, G. H. Bernstein, "High-Resolution Electron Beam Lithography and DNA Nano-Patterning for Molecular QCA", IEEE Transactions on Nanotechnology, vol. 4 no. 3, pp. 312-316, 2005. 